Supporting Information

\title{
Application of conductance study to analyze micellization behavior of cationic gemini surfactants in water and water - ethanol solvent mixed media
}

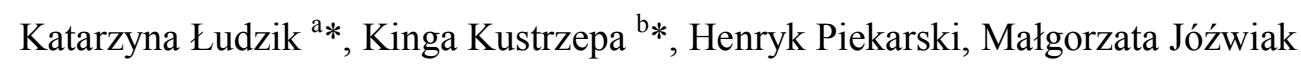

University of Łódź, Faculty of Chemistry, Department of Physical Chemistry, Pomorska 165, 90-236 Łódź, Poland

AUTHOR INFORMATION

*Corresponding Authors

${ }^{a}$ E-mail: kasialudzik@tlen.pl

${ }^{\mathrm{b}}$ E-mail: kakus@uni.lodz.pl 


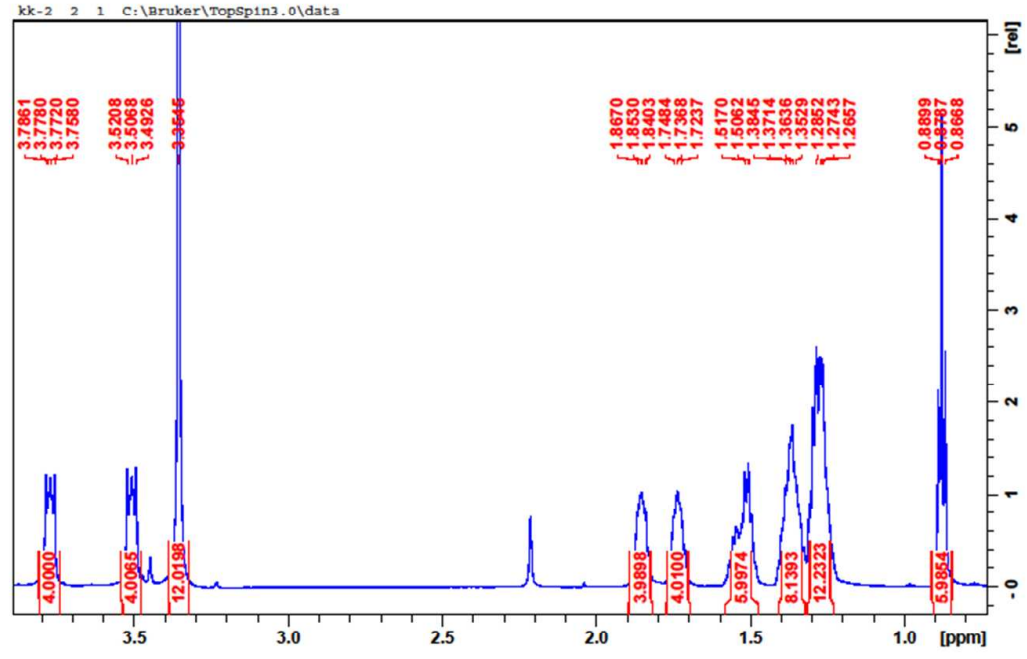

${ }^{1} \mathrm{H}-\mathrm{NMR}\left(600 \mathrm{MHz}, \mathrm{CDCl}_{3}\right): \delta=0,88\left(\mathrm{t}, 6 \mathrm{H}, J=6,7 \mathrm{~Hz}, 2 \mathrm{CH}_{3}\right), 1,22-1,31\left(\mathrm{~m}, 12 \mathrm{H}, 6 \mathrm{CH}_{2}\right), 1,31-1,41(\mathrm{~m}, 8 \mathrm{H}$, $\left.4 \mathrm{CH}_{2}\right), 1,47-1,58\left(\mathrm{~m}, 6 \mathrm{H}, 3 \mathrm{CH}_{2}\right), 1,70-1.77\left(\mathrm{~m}, 4 \mathrm{H}, 2 \mathrm{CH}_{2}\right), 1,82-1.89\left(\mathrm{~m}, 4 \mathrm{H}, 2 \mathrm{CH}_{2}\right), 3,35\left(\mathrm{~s}, 12 \mathrm{H}, 4 \mathrm{CH}_{3} \mathrm{~N}\right)$, $3,48-3,54\left(\mathrm{~m}, 4 \mathrm{H}, 2 \mathrm{CH}_{2} \mathrm{~N}\right), 3,74-3.81\left(\mathrm{~m}, 4 \mathrm{H}, 2 \mathrm{CH}_{2} \mathrm{~N}\right)$.

Figure S1. ${ }^{1}$ H-NMR Spectra of heptylene-1,7-bis(dimethyloctylammonium) bromide (8-78)

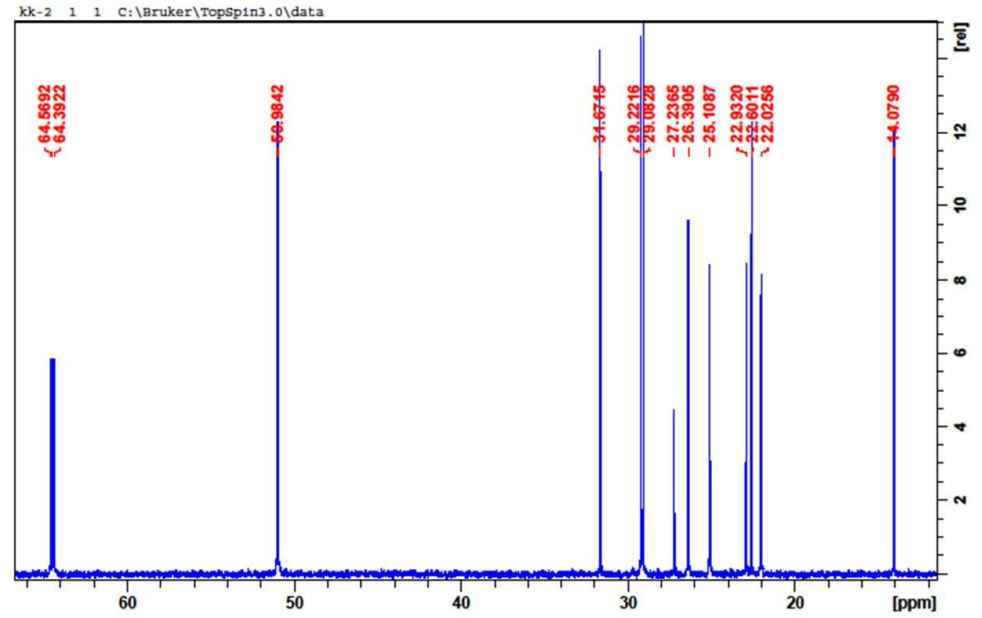

${ }^{13} \mathrm{C}-\mathrm{NMR}\left(150 \mathrm{MHz}, \mathrm{CDCl}_{3}\right): \delta=14,08\left(2 \mathrm{CH}_{3}\right), 22,03 ; 22,60 ; 22,93 ; 25,11 ; 26,39 ; 27,23 ; 29,08 ; 29,22 ; 31,67$ $\left(17 \mathrm{CH}_{2}\right), 50,98\left(4 \mathrm{CH}_{3} \mathrm{~N}\right), 64,39\left(2 \mathrm{CH}_{2} \mathrm{~N}\right), 64,57\left(2 \mathrm{CH}_{2} \mathrm{~N}\right)$.

Figure S2. ${ }^{13}$ C-NMR Spectra of heptylene-1,7-bis(dimethyloctylammonium) bromide (8-78) 


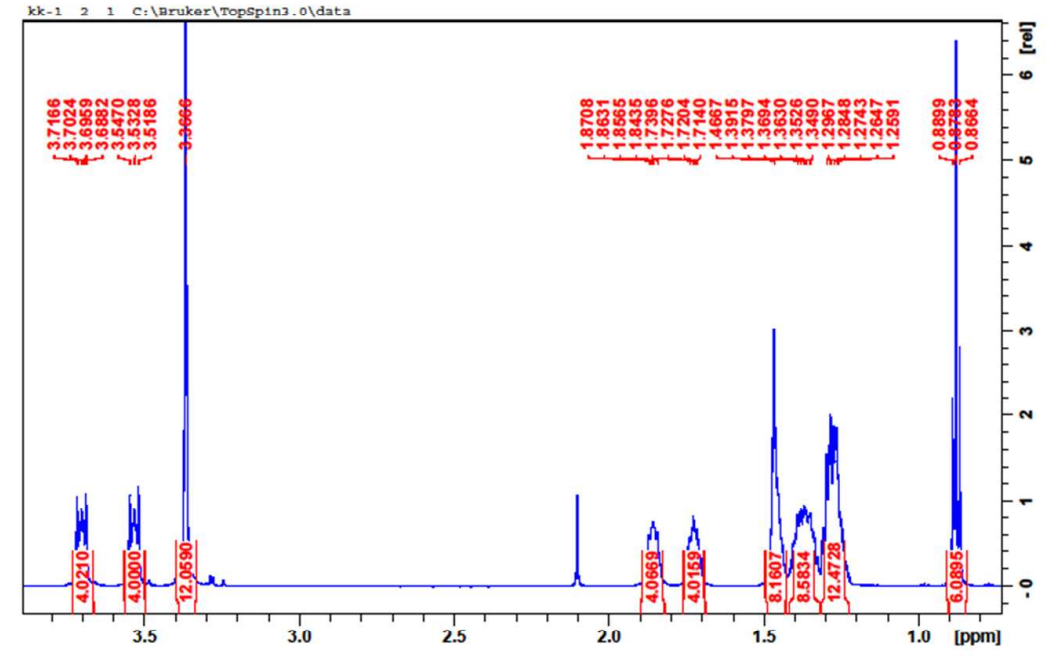

${ }^{1} \mathrm{H}-\mathrm{NMR}\left(600 \mathrm{MHz}, \mathrm{CDCl}_{3}\right) \delta=0,88\left(\mathrm{t}, 6 \mathrm{H}, \mathrm{J}=7,0 \mathrm{~Hz}, 2 \mathrm{CH}_{3}\right), 1,22-1,32(\mathrm{~m}, 12 \mathrm{H}, 6 \mathrm{CH}), 1,32-1,42(\mathrm{~m}, 8 \mathrm{H}$, $\left.4 \mathrm{CH}_{2}\right), 1,43-1,50\left(\mathrm{~m}, 8 \mathrm{H}, 4 \mathrm{CH}_{2}\right), 1,69-1,75\left(\mathrm{~m}, 4 \mathrm{H}, 2 \mathrm{CH}_{2}\right), 1,83-1,89\left(\mathrm{~m}, 4 \mathrm{H}, 2 \mathrm{CH}_{2}\right), 3,37\left(\mathrm{~s}, 12 \mathrm{H}, 4 \mathrm{CH}_{3} \mathrm{~N}\right)$, $3,50-3,56\left(\mathrm{~m}, 4 \mathrm{H}, 2 \mathrm{CH}_{2} \mathrm{~N}\right), 3,66-3,74\left(\mathrm{~m}, 4 \mathrm{H}, 2 \mathrm{CH}_{2} \mathrm{~N}\right)$.

Figure S3. ${ }^{1}$ H-NMR Spectra of octylene-1,8-bis(dimethyloctylammonium) bromide (8-8-8)

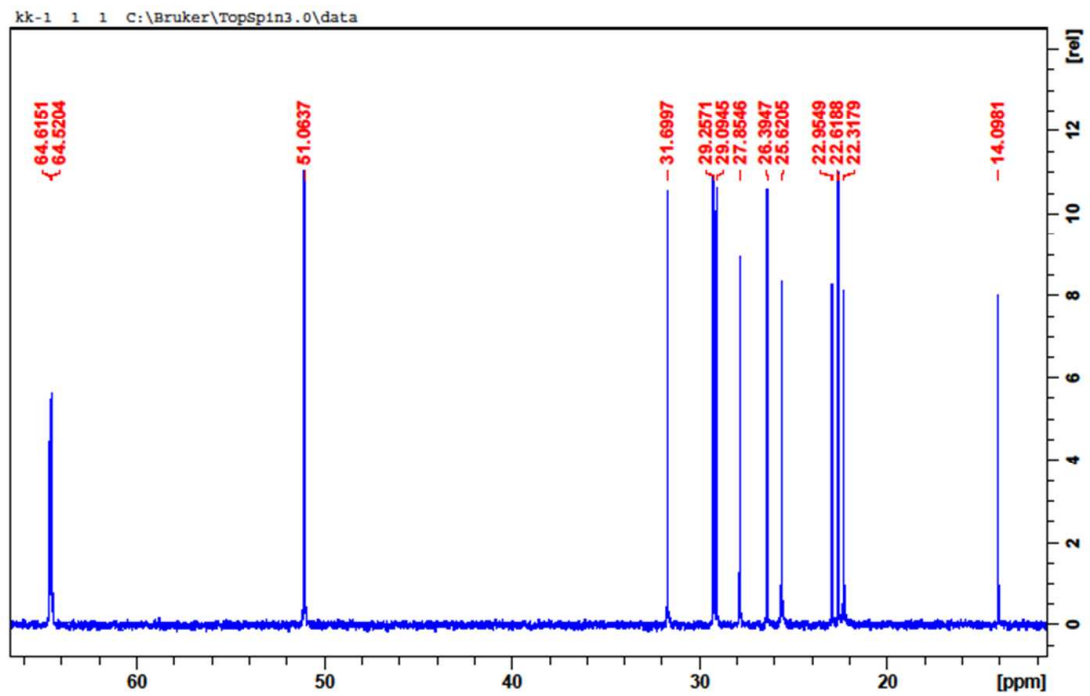

${ }^{13} \mathrm{C}-\mathrm{NMR}\left(150 \mathrm{MHz}, \mathrm{CDCl}_{3}\right) \delta=14,09\left(2 \mathrm{CH}_{3}\right), 22,32 ; 22,62 ; 22,95 ; 25,62 ; 26,39 ; 27,85 ; 29,09 ; 29,25 ; 31,70$ $\left(18 \mathrm{CH}_{2}\right) ; 51,06\left(4 \mathrm{CH}_{3} \mathrm{~N}\right) ; 64,52\left(2 \mathrm{CH}_{2} \mathrm{~N}\right) ; 64,62\left(2 \mathrm{CH}_{2} \mathrm{~N}\right)$.

Figure S4. ${ }^{13}$ C-NMR Spectra of octylene-1,8-bis(dimethyloctylammonium) bromide (8-8-8) 
Table S1. Values of conductivity $\kappa$ for different concentration $-m$ of aqueous solutions of 8-7-8 surfactants within $288.15-303.15 \mathrm{~K}$ temperature range $(p=0.1 \mathrm{MPa})$.

\begin{tabular}{|c|c|c|c|c|c|c|c|}
\hline \multicolumn{2}{|c|}{$288.15 \mathrm{~K}$} & \multicolumn{2}{|c|}{$293.15 \mathrm{~K}$} & \multicolumn{2}{|c|}{$298.15 \mathrm{~K}$} & \multicolumn{2}{|c|}{$303.15 \mathrm{~K}$} \\
\hline$m / \mathrm{mol} \cdot \mathrm{kg}^{-1}$ & $\kappa / \mathrm{S} \cdot \mathrm{m}^{-1}$ & $m / \mathrm{mol} \cdot \mathrm{kg}^{-1}$ & $\kappa / \mathrm{S} \cdot \mathrm{m}^{-1}$ & $m / \mathrm{mol} \cdot \mathrm{kg}^{-1}$ & $\kappa / \mathrm{S} \cdot \mathrm{m}^{-1}$ & $m / \mathrm{mol} \cdot \mathrm{kg}^{-1}$ & $\kappa / \mathrm{S} \cdot \mathrm{m}^{-1}$ \\
\hline 0.00346 & 0.0450 & 0.00358 & 0.0614 & 0.00412 & 0.0733 & 0.00395 & 0.0825 \\
\hline 0.00683 & 0.0906 & 0.00707 & 0.1141 & 0.00813 & 0.1367 & 0.00780 & 0.1546 \\
\hline 0.01013 & 0.1321 & 0.01048 & 0.1619 & 0.01203 & 0.1940 & 0.01155 & 0.2192 \\
\hline 0.01335 & 0.1701 & 0.01381 & 0.2059 & 0.01584 & 0.2465 & 0.01522 & 0.2786 \\
\hline 0.01649 & 0.2053 & 0.01706 & 0.2467 & 0.01956 & 0.2953 & 0.01880 & 0.3341 \\
\hline 0.01957 & 0.2383 & 0.02023 & 0.2847 & 0.02318 & 0.3409 & 0.02229 & 0.3857 \\
\hline 0.02257 & 0.2693 & 0.02333 & 0.3205 & 0.02672 & 0.3836 & 0.02571 & 0.4344 \\
\hline 0.02551 & 0.2984 & 0.02635 & 0.3542 & 0.03018 & 0.4238 & 0.02904 & 0.4801 \\
\hline 0.02839 & 0.3261 & 0.02931 & 0.3863 & 0.03355 & 0.4622 & 0.03230 & 0.5234 \\
\hline 0.03120 & 0.3525 & 0.03221 & 0.4164 & 0.03684 & 0.4985 & 0.03549 & 0.5642 \\
\hline 0.03396 & 0.3773 & 0.03504 & 0.4453 & 0.04006 & 0.5328 & 0.03860 & 0.6027 \\
\hline 0.03665 & 0.4012 & 0.03780 & 0.4726 & 0.04320 & 0.5664 & 0.04165 & 0.6395 \\
\hline 0.03929 & 0.4237 & 0.04051 & 0.4989 & 0.04628 & 0.5975 & 0.04463 & 0.6742 \\
\hline 0.04187 & 0.4456 & 0.04316 & 0.5237 & 0.04928 & 0.6276 & 0.04755 & 0.7070 \\
\hline 0.04440 & 0.4610 & 0.04576 & 0.5474 & 0.05222 & 0.6556 & 0.05040 & 0.7370 \\
\hline 0.04688 & 0.4806 & 0.04830 & 0.5700 & 0.05510 & 0.6806 & 0.05320 & 0.7654 \\
\hline 0.04931 & 0.5009 & 0.05078 & 0.5891 & 0.05791 & 0.7052 & 0.05594 & 0.7928 \\
\hline 0.05169 & 0.5198 & 0.05322 & 0.6085 & 0.06066 & 0.7286 & 0.05862 & 0.8182 \\
\hline 0.05403 & 0.5340 & 0.05561 & 0.6274 & 0.06336 & 0.7512 & 0.06124 & 0.8422 \\
\hline 0.05632 & 0.5494 & 0.05795 & 0.6459 & 0.06600 & 0.7722 & 0.06382 & 0.8647 \\
\hline 0.05856 & 0.5647 & 0.06024 & 0.6632 & 0.06858 & 0.7917 & 0.06634 & 0.8857 \\
\hline 0.06076 & 0.5807 & 0.06249 & 0.6801 & 0.07111 & 0.8100 & 0.06881 & 0.9055 \\
\hline 0.06292 & 0.5961 & 0.06469 & 0.6958 & 0.07359 & 0.8269 & 0.07123 & 0.9241 \\
\hline 0.06504 & 0.6110 & 0.06685 & 0.7104 & 0.07602 & 0.8426 & 0.07361 & 0.9413 \\
\hline 0.06712 & 0.6248 & 0.06897 & 0.7244 & 0.07841 & 0.8573 & 0.07594 & 0.9574 \\
\hline 0.06916 & 0.6378 & 0.07105 & 0.7376 & 0.08074 & 0.8710 & 0.07823 & 0.9726 \\
\hline 0.07117 & 0.6503 & 0.07309 & 0.7496 & 0.08303 & 0.8838 & 0.08047 & 0.9867 \\
\hline 0.07313 & 0.6618 & 0.07510 & 0.7611 & 0.08528 & 0.8958 & 0.08267 & 1.0004 \\
\hline 0.07507 & 0.6728 & 0.07706 & 0.7718 & 0.08748 & 0.9072 & 0.08483 & 1.0133 \\
\hline 0.07697 & 0.6825 & 0.07899 & 0.7823 & 0.08964 & 0.9180 & 0.08695 & 1.0258 \\
\hline 0.07883 & 0.6918 & 0.08089 & 0.7922 & 0.09176 & 0.9283 & 0.08904 & 1.0377 \\
\hline 0.08066 & 0.7006 & 0.08275 & 0.8007 & 0.09385 & 0.9381 & 0.09108 & 1.0486 \\
\hline 0.08246 & 0.7086 & 0.08458 & 0.8095 & 0.09589 & 0.9474 & 0.09309 & 1.0594 \\
\hline 0.08423 & 0.7159 & 0.08637 & 0.8177 & 0.09790 & 0.9564 & 0.09506 & 1.0699 \\
\hline 0.08597 & 0.7231 & 0.08814 & 0.8255 & 0.09987 & 0.9652 & 0.09700 & 1.0799 \\
\hline 0.08768 & 0.7297 & 0.08987 & 0.8330 & 0.10180 & 0.9737 & 0.09891 & 1.0894 \\
\hline
\end{tabular}


Table S1. continuation. Values of conductivity $\kappa$ for different concentration $-m$ of aqueous solutions of 8-7-8 surfactants within 288.15 - 303.15 $\mathrm{K}$ temperature range $(p=0.1 \mathrm{MPa})$.

\begin{tabular}{|c|c|c|c|c|c|c|c|}
\hline \multicolumn{2}{|c|}{$288.15 \mathrm{~K}$} & \multicolumn{2}{|c|}{$293.15 \mathrm{~K}$} & \multicolumn{2}{|c|}{$298.15 \mathrm{~K}$} & \multicolumn{2}{|c|}{$303.15 \mathrm{~K}$} \\
\hline$m / \mathrm{mol} \cdot \mathrm{kg}^{-1}$ & $\kappa / S \cdot \mathrm{m}^{-1}$ & $m / \mathrm{mol} \cdot \mathrm{kg}^{-1}$ & $\kappa / \mathrm{S} \cdot \mathrm{m}^{-1}$ & $m / \mathrm{mol} \cdot \mathrm{kg}^{-1}$ & $\kappa / S \cdot \mathrm{m}^{-1}$ & $m / \mathrm{mol} \cdot \mathrm{kg}^{-1}$ & $\kappa / S \cdot \mathrm{m}^{-1}$ \\
\hline 0.08936 & 0.7375 & 0.09157 & 0.8399 & 0.10370 & 0.9813 & 0.10078 & 1.0989 \\
\hline 0.09102 & 0.7433 & 0.09325 & 0.8467 & 0.10557 & 0.9894 & 0.10262 & 1.1081 \\
\hline 0.09264 & 0.7486 & 0.09490 & 0.8531 & 0.10741 & 0.9968 & 0.10443 & 1.1172 \\
\hline 0.09424 & 0.7533 & 0.09652 & 0.8596 & 0.10921 & 1.0040 & 0.10621 & 1.1254 \\
\hline 0.09582 & 0.7584 & 0.09811 & 0.8656 & 0.11098 & 1.0113 & 0.10795 & 1.1341 \\
\hline 0.09737 & 0.7635 & 0.09967 & 0.8712 & 0.11272 & 1.0180 & 0.10968 & 1.1428 \\
\hline 0.09889 & 0.7686 & 0.10122 & 0.8769 & 0.11444 & 1.0247 & 0.11137 & 1.1494 \\
\hline 0.10039 & 0.7735 & 0.10273 & 0.8822 & 0.11612 & 1.0312 & 0.11303 & 1.1525 \\
\hline 0.10187 & 0.7782 & 0.10422 & 0.8877 & 0.11778 & 1.0376 & 0.11467 & 1.1586 \\
\hline 0.10332 & 0.7828 & 0.10569 & 0.8931 & 0.11941 & 1.0437 & 0.11628 & 1.1652 \\
\hline 0.10475 & 0.7873 & 0.10714 & 0.8979 & 0.12101 & 1.0501 & 0.11787 & 1.1723 \\
\hline 0.10616 & 0.7914 & 0.10856 & 0.9028 & 0.12259 & 1.0561 & 0.11943 & 1.1795 \\
\hline 0.10755 & 0.7956 & 0.10996 & 0.9078 & 0.12414 & 1.0618 & 0.12096 & 1.1864 \\
\hline 0.10891 & 0.7996 & 0.11134 & 0.9126 & 0.12566 & 1.0676 & 0.12248 & 1.1934 \\
\hline 0.11026 & 0.8036 & 0.11269 & 0.9169 & 0.12717 & 1.0730 & 0.12397 & 1.1999 \\
\hline 0.11158 & 0.8074 & 0.11403 & 0.9214 & 0.12865 & 1.0784 & 0.12543 & 1.2068 \\
\hline 0.11289 & 0.8111 & 0.11535 & 0.9255 & 0.13010 & 1.0836 & 0.12688 & 1.2133 \\
\hline 0.11418 & 0.8148 & 0.11664 & 0.9298 & 0.13154 & 1.0886 & 0.12830 & 1.2196 \\
\hline 0.11545 & 0.8184 & 0.11792 & 0.9337 & 0.13295 & 1.0937 & 0.12970 & 1.2253 \\
\hline 0.11670 & 0.8218 & 0.11918 & 0.9381 & 0.13434 & 1.0987 & 0.13108 & 1.2314 \\
\hline 0.11793 & 0.8250 & 0.12042 & 0.9422 & 0.13571 & 1.1034 & 0.13245 & 1.2375 \\
\hline 0.11914 & 0.8283 & 0.12164 & 0.9463 & 0.13706 & 1.1083 & 0.13379 & 1.2434 \\
\hline 0.12034 & 0.8317 & 0.12285 & 0.9498 & 0.13839 & 1.1131 & 0.13511 & 1.2489 \\
\hline 0.12152 & 0.8351 & 0.12403 & 0.9537 & 0.13970 & 1.1176 & 0.13641 & 1.2544 \\
\hline
\end{tabular}

Standard uncertainties are: $u(\mathrm{~T})=0.1 \mathrm{~K}, u(\mathrm{~m})=10^{-5} \mathrm{~m}, u(p)=0.05 p$ and the combined expanded uncertainty $U_{c}(\kappa)=0.004 \kappa($ level of confidence $=0.95)$. 
Table S2. Values of conductivity $\kappa$ for different concentration $-m$ of aqueous solutions of 8-7-8 surfactants within $308.15-323.15 \mathrm{~K}$ temperature range $(p=0.1 \mathrm{MPa})$.

\begin{tabular}{|c|c|c|c|c|c|c|c|}
\hline \multicolumn{2}{|c|}{$308.15 \mathrm{~K}$} & \multicolumn{2}{|c|}{$313.15 \mathrm{~K}$} & \multicolumn{2}{|c|}{$318.15 \mathrm{~K}$} & \multicolumn{2}{|c|}{$323.15 \mathrm{~K}$} \\
\hline$m / \mathrm{mol} \cdot \mathrm{kg}^{-1}$ & $\kappa / \mathrm{S} \cdot \mathrm{m}^{-1}$ & $m / \mathrm{mol} \cdot \mathrm{kg}^{-1}$ & $\kappa / \mathrm{S} \cdot \mathrm{m}^{-1}$ & $m / \mathrm{mol} \cdot \mathrm{kg}^{-1}$ & $\kappa / \mathrm{S} \cdot \mathrm{m}^{-1}$ & $m / \mathrm{mol} \cdot \mathrm{kg}^{-1}$ & $\kappa / \mathrm{S} \cdot \mathrm{m}^{-1}$ \\
\hline 0.00314 & 0.0748 & 0.00321 & 0.0867 & 0.00275 & 0.0804 & 0.00277 & 0.0873 \\
\hline 0.00620 & 0.1404 & 0.00651 & 0.1626 & 0.00557 & 0.1507 & 0.00548 & 0.1644 \\
\hline 0.00919 & 0.2000 & 0.00972 & 0.2318 & 0.00825 & 0.2156 & 0.00812 & 0.2351 \\
\hline 0.01211 & 0.2553 & 0.01285 & 0.2956 & 0.01087 & 0.2766 & 0.01069 & 0.3008 \\
\hline 0.01497 & 0.3068 & 0.01590 & 0.3549 & 0.01343 & 0.3337 & 0.01321 & 0.3629 \\
\hline 0.01775 & 0.3553 & 0.01887 & 0.4110 & 0.01593 & 0.3879 & 0.01566 & 0.4212 \\
\hline 0.02047 & 0.4012 & 0.02177 & 0.4636 & 0.01837 & 0.4391 & 0.01806 & 0.4754 \\
\hline 0.02313 & 0.4441 & 0.02460 & 0.5134 & 0.02075 & 0.4875 & 0.02041 & 0.5277 \\
\hline 0.02574 & 0.4852 & 0.02737 & 0.5608 & 0.02309 & 0.5336 & 0.02270 & 0.5778 \\
\hline 0.02828 & 0.5247 & 0.03007 & 0.6060 & 0.02536 & 0.5768 & 0.02494 & 0.6254 \\
\hline 0.03077 & 0.5621 & 0.03270 & 0.6486 & 0.02759 & 0.6191 & 0.02712 & 0.6715 \\
\hline 0.03320 & 0.5977 & 0.03528 & 0.6897 & 0.02977 & 0.6595 & 0.02926 & 0.7138 \\
\hline 0.03558 & 0.6323 & 0.03779 & 0.7286 & 0.03191 & 0.6992 & 0.03136 & 0.7530 \\
\hline 0.03792 & 0.6661 & 0.04025 & 0.7657 & 0.03400 & 0.7358 & 0.03341 & 0.7921 \\
\hline 0.04020 & 0.6997 & 0.04266 & 0.8008 & 0.03604 & 0.7720 & 0.03541 & 0.8301 \\
\hline 0.04243 & 0.7310 & 0.04501 & 0.8346 & 0.03804 & 0.8061 & 0.03738 & 0.8645 \\
\hline 0.04462 & 0.7591 & 0.04731 & 0.8666 & 0.04000 & 0.8387 & 0.03930 & 0.8986 \\
\hline 0.04677 & 0.7860 & 0.04956 & 0.8969 & 0.04192 & 0.8696 & 0.04118 & 0.9304 \\
\hline 0.04887 & 0.8115 & 0.05176 & 0.9261 & 0.04380 & 0.8989 & 0.04303 & 0.9603 \\
\hline 0.05093 & 0.8363 & 0.05392 & 0.9541 & 0.04564 & 0.9262 & 0.04483 & 0.9926 \\
\hline 0.05295 & 0.8600 & 0.05603 & 0.9819 & 0.04745 & 0.9522 & 0.04660 & 1.0218 \\
\hline 0.05493 & 0.8824 & 0.05810 & 1.0077 & 0.04922 & 0.9784 & 0.04834 & 1.0494 \\
\hline 0.05688 & 0.9035 & 0.06013 & 1.0320 & 0.05096 & 1.0043 & 0.05004 & 1.0774 \\
\hline 0.05878 & 0.9239 & 0.06211 & 1.0546 & 0.05266 & 1.0258 & 0.05171 & 1.1042 \\
\hline 0.06065 & 0.9434 & 0.06405 & 1.0757 & 0.05433 & 1.0487 & 0.05335 & 1.1307 \\
\hline 0.06248 & 0.9619 & 0.06596 & 1.0961 & 0.05597 & 1.0710 & 0.05495 & 1.1552 \\
\hline 0.06428 & 0.9790 & 0.06783 & 1.1153 & 0.05757 & 1.0923 & 0.05653 & 1.1791 \\
\hline 0.06605 & 0.9956 & 0.06966 & 1.1338 & 0.05915 & 1.1133 & 0.05808 & 1.2030 \\
\hline 0.06778 & 1.0114 & 0.07146 & 1.1510 & 0.06070 & 1.1328 & 0.05959 & 1.2231 \\
\hline 0.06949 & 1.0263 & 0.07322 & 1.1678 & 0.06222 & 1.1506 & 0.06108 & 1.2446 \\
\hline 0.07116 & 1.0404 & 0.07495 & 1.1836 & 0.06372 & 1.1656 & 0.06254 & 1.2654 \\
\hline 0.07280 & 1.0542 & 0.07665 & 1.1981 & 0.06518 & 1.1858 & 0.06398 & 1.2833 \\
\hline 0.07442 & 1.0668 & 0.07831 & 1.2127 & 0.06662 & 1.1977 & 0.06539 & 1.2997 \\
\hline 0.07600 & 1.0790 & 0.07994 & 1.2263 & 0.06804 & 1.2143 & 0.06678 & 1.3198 \\
\hline 0.07756 & 1.0898 & 0.08155 & 1.2399 & 0.06943 & 1.2281 & 0.06814 & 1.3330 \\
\hline 0.07909 & 1.1022 & 0.08313 & 1.2527 & 0.07079 & 1.2444 & 0.06948 & 1.3491 \\
\hline
\end{tabular}


Table S2. continuation. Values of conductivity $\kappa$ for different concentration $-m$ of aqueous solutions of 8-7-8 surfactants within 308.15 - 323.15 K temperature range $(p=$ 0.1 MPa).

\begin{tabular}{|c|c|c|c|c|c|c|c|}
\hline \multicolumn{2}{|c|}{$308.15 \mathrm{~K}$} & \multicolumn{2}{|c|}{$313.15 \mathrm{~K}$} & \multicolumn{2}{|c|}{$318.15 \mathrm{~K}$} & \multicolumn{2}{|c|}{$323.15 \mathrm{~K}$} \\
\hline$m / \mathrm{mol} \cdot \mathrm{kg}^{-1}$ & $\kappa / S \cdot m^{-1}$ & $m / \mathrm{mol} \cdot \mathrm{kg}^{-1}$ & $\kappa / S \cdot m^{-1}$ & $\mathrm{~m} / \mathrm{mol} \cdot \mathrm{kg}^{-1}$ & $\kappa / S \cdot m^{-1}$ & $m / \mathrm{mol} \cdot \mathrm{kg}^{-1}$ & $\kappa / S \cdot m^{-1}$ \\
\hline 0.08060 & 1.1118 & 0.08467 & 1.2647 & 0.07214 & 1.2569 & 0.07079 & 1.3645 \\
\hline 0.08208 & 1.1219 & 0.08619 & 1.2764 & 0.07346 & 1.2706 & 0.07208 & 1.3798 \\
\hline 0.08353 & 1.1327 & 0.08769 & 1.2879 & 0.07476 & 1.2841 & 0.07335 & 1.3942 \\
\hline 0.08496 & 1.1426 & 0.08915 & 1.2981 & 0.07603 & 1.2963 & 0.07460 & 1.4084 \\
\hline 0.08637 & 1.1523 & 0.09060 & 1.3086 & 0.07729 & 1.3096 & 0.07583 & 1.4220 \\
\hline 0.08776 & 1.1621 & 0.09201 & 1.3189 & 0.07852 & 1.3200 & 0.07704 & 1.4358 \\
\hline 0.08912 & 1.1712 & 0.09341 & 1.3268 & 0.07974 & 1.3335 & 0.07822 & 1.4480 \\
\hline 0.09046 & 1.1795 & 0.09478 & 1.3358 & 0.08093 & 1.3436 & 0.07939 & 1.4603 \\
\hline 0.09178 & 1.1886 & 0.09612 & 1.3449 & 0.08211 & 1.3557 & 0.08054 & 1.4722 \\
\hline 0.09307 & 1.1971 & 0.09745 & 1.3540 & 0.08326 & 1.3650 & 0.08167 & 1.4847 \\
\hline 0.09435 & 1.2050 & 0.09875 & 1.3630 & 0.08440 & 1.3763 & 0.08279 & 1.4945 \\
\hline 0.09561 & 1.2127 & 0.10003 & 1.3693 & 0.08552 & 1.3851 & 0.08388 & 1.5060 \\
\hline 0.09685 & 1.2203 & 0.10129 & 1.3783 & 0.08663 & 1.3953 & 0.08496 & 1.5150 \\
\hline 0.09807 & 1.2289 & 0.10253 & 1.3855 & 0.08771 & 1.4033 & 0.08602 & 1.5260 \\
\hline 0.09927 & 1.2361 & 0.10375 & 1.3923 & 0.08878 & 1.4127 & 0.08707 & 1.5336 \\
\hline 0.10045 & 1.2436 & 0.10495 & 1.3989 & 0.08984 & 1.4208 & 0.08810 & 1.5423 \\
\hline 0.10162 & 1.2506 & 0.10614 & 1.4051 & 0.09087 & 1.4284 & 0.08911 & 1.5504 \\
\hline 0.10276 & 1.2573 & 0.10730 & 1.4110 & 0.09190 & 1.4381 & 0.09011 & 1.5581 \\
\hline 0.10389 & 1.2637 & 0.10845 & 1.4165 & 0.09290 & 1.4425 & 0.09109 & 1.5654 \\
\hline 0.10501 & 1.2721 & 0.10958 & 1.4217 & 0.09390 & 1.4489 & 0.09206 & 1.5722 \\
\hline 0.10611 & 1.2783 & 0.11069 & 1.4266 & 0.09487 & 1.4549 & 0.09302 & 1.5785 \\
\hline 0.10719 & 1.2843 & 0.11178 & 1.4312 & 0.09584 & 1.4597 & 0.09396 & 1.5844 \\
\hline 0.10826 & 1.2901 & 0.11286 & 1.4355 & 0.09679 & 1.4657 & 0.09489 & 1.5898 \\
\hline
\end{tabular}

Standard uncertainties are: $u(\mathrm{~T})=0.1 K, u(\mathrm{~m})=10^{-5} \mathrm{~m}, u(p)=0.05 p$ and the combined expanded uncertainty $U_{c}(\kappa)=0.004 \kappa($ level of confidence $=0.95)$. 
Table S3. Values of conductivity $\kappa$ for different concentration $-m$ of 8-7-8 surfactant in $10 \mathrm{wt} \%$ ethanol-water mixtures within $288.15-303.15 \mathrm{~K}$ temperature range $(p=0.1$ MPa).

\begin{tabular}{|c|c|c|c|c|c|c|c|}
\hline \multicolumn{2}{|c|}{$288.15 \mathrm{~K}$} & \multicolumn{2}{|c|}{$293.15 \mathrm{~K}$} & \multicolumn{2}{|c|}{$298.15 \mathrm{~K}$} & \multicolumn{2}{|c|}{$303.15 \mathrm{~K}$} \\
\hline$m / \mathrm{mol} \cdot \mathrm{kg}^{-1}$ & $\kappa / \mathrm{S} \cdot \mathrm{m}^{-1}$ & $m / \mathrm{mol} \cdot \mathrm{kg}^{-1}$ & $\kappa / \mathrm{S} \cdot \mathrm{m}^{-1}$ & $m / \mathrm{mol}^{\prime} \mathrm{kg}^{-1}$ & $\kappa / \mathrm{S} \cdot \mathrm{m}^{-1}$ & $m / \mathrm{mol}^{\prime} \mathrm{kg}^{-1}$ & $\kappa / \mathrm{S} \cdot \mathrm{m}^{-1}$ \\
\hline 0.00363 & 0.0393 & 0.00361 & 0.0476 & 0.00280 & 0.0424 & 0.00367 & 0.0641 \\
\hline 0.00722 & 0.0744 & 0.00716 & 0.0869 & 0.00556 & 0.0811 & 0.00728 & 0.1167 \\
\hline 0.01075 & 0.1064 & 0.01067 & 0.1243 & 0.00829 & 0.1164 & 0.01084 & 0.1665 \\
\hline 0.01424 & 0.1365 & 0.01413 & 0.1597 & 0.01098 & 0.1496 & 0.01436 & 0.2136 \\
\hline 0.01768 & 0.1644 & 0.01754 & 0.1933 & 0.01364 & 0.1809 & 0.01783 & 0.2583 \\
\hline 0.02107 & 0.1909 & 0.02091 & 0.2253 & 0.01626 & 0.2104 & 0.02125 & 0.3005 \\
\hline 0.02442 & 0.2162 & 0.02423 & 0.2556 & 0.01884 & 0.2389 & 0.02462 & 0.3404 \\
\hline 0.02773 & 0.2403 & 0.02751 & 0.2843 & 0.02139 & 0.2659 & 0.02794 & 0.3782 \\
\hline 0.03099 & 0.2630 & 0.03075 & 0.3115 & 0.02391 & 0.2919 & 0.03123 & 0.4139 \\
\hline 0.03420 & 0.2847 & 0.03394 & 0.3373 & 0.02640 & 0.3168 & 0.03447 & 0.4476 \\
\hline 0.03738 & 0.3056 & 0.03709 & 0.3618 & 0.02886 & 0.3407 & 0.03767 & 0.4795 \\
\hline 0.04051 & 0.3258 & 0.04328 & 0.4070 & 0.03128 & 0.3637 & 0.04083 & 0.5097 \\
\hline 0.04361 & 0.3452 & 0.04631 & 0.4278 & 0.03368 & 0.3860 & 0.04394 & 0.5382 \\
\hline 0.04666 & 0.3637 & 0.04931 & 0.4476 & 0.03604 & 0.4074 & 0.04702 & 0.5652 \\
\hline 0.04967 & 0.3813 & 0.05518 & 0.4842 & 0.03838 & 0.4280 & 0.05005 & 0.5908 \\
\hline 0.05265 & 0.3980 & 0.05807 & 0.5011 & 0.04069 & 0.4478 & 0.05305 & 0.6150 \\
\hline 0.05559 & 0.4141 & 0.06092 & 0.5171 & 0.04297 & 0.4670 & 0.05601 & 0.6379 \\
\hline 0.05850 & 0.4294 & 0.06373 & 0.5324 & 0.04522 & 0.4854 & 0.05893 & 0.6597 \\
\hline 0.06136 & 0.4427 & 0.06848 & 0.5570 & 0.04744 & 0.5031 & 0.06182 & 0.6803 \\
\hline 0.06419 & 0.4556 & 0.07313 & 0.5795 & 0.04964 & 0.5200 & 0.06467 & 0.7000 \\
\hline 0.06897 & 0.4769 & 0.07769 & 0.6003 & 0.05368 & 0.5487 & 0.06948 & 0.7314 \\
\hline 0.07365 & 0.4961 & 0.08215 & 0.6196 & 0.05763 & 0.5755 & 0.07419 & 0.7604 \\
\hline 0.07824 & 0.5134 & 0.08653 & 0.6375 & 0.06149 & 0.6001 & 0.07880 & 0.7872 \\
\hline 0.08273 & 0.5292 & 0.09082 & 0.6542 & 0.06528 & 0.6232 & 0.08331 & 0.8121 \\
\hline 0.08713 & 0.5437 & 0.09503 & 0.6699 & 0.06898 & 0.6446 & 0.08774 & 0.8354 \\
\hline 0.09144 & 0.5573 & 0.09915 & 0.6846 & 0.07260 & 0.6643 & 0.09208 & 0.8572 \\
\hline 0.09567 & 0.5700 & 0.10320 & 0.6985 & 0.07615 & 0.6829 & 0.09633 & 0.8777 \\
\hline 0.09981 & 0.5819 & 0.10716 & 0.7116 & 0.07963 & 0.7002 & 0.10050 & 0.8972 \\
\hline 0.10388 & 0.5933 & 0.11106 & 0.7242 & 0.08303 & 0.7166 & 0.10458 & 0.9157 \\
\hline 0.10787 & 0.6040 & 0.11488 & 0.7361 & 0.08637 & 0.7322 & 0.10859 & 0.9334 \\
\hline 0.11178 & 0.6145 & 0.11863 & 0.7476 & 0.08964 & 0.7468 & 0.11252 & 0.9504 \\
\hline 0.11562 & 0.6245 & 0.12231 & 0.7587 & 0.09284 & 0.7605 & 0.11638 & 0.9668 \\
\hline 0.11939 & 0.6339 & 0.12593 & 0.7694 & 0.09599 & 0.7738 & 0.12017 & 0.9826 \\
\hline 0.12309 & 0.6431 & 0.12948 & 0.7797 & 0.09907 & 0.7866 & 0.12388 & 0.9980 \\
\hline 0.12672 & 0.6522 & 0.13297 & 0.7898 & 0.10209 & 0.7987 & 0.12753 & 1.0129 \\
\hline 0.13029 & 0.6606 & 0.13640 & 0.7996 & 0.10505 & 0.8104 & 0.13111 & 1.0274 \\
\hline
\end{tabular}


Table S3. continuation. Values of conductivity $\kappa$ for different concentration $-m$ of 8-78 surfactant in $10 \mathrm{wt} \%$ ethanol-water mixtures within $288.15-303.15 \mathrm{~K}$ temperature range $(p=0.1 \mathrm{MPa})$.

\begin{tabular}{|c|c|c|c|c|c|c|c|}
\hline \multicolumn{2}{|c|}{$288.15 \mathrm{~K}$} & \multicolumn{2}{|c|}{$293.15 \mathrm{~K}$} & \multicolumn{2}{|c|}{$298.15 \mathrm{~K}$} & \multicolumn{2}{|c|}{$303.15 \mathrm{~K}$} \\
\hline$m / \mathrm{mol} \cdot \mathrm{kg}^{-1}$ & $\kappa / \mathrm{S} \cdot \mathrm{m}^{-1}$ & $m / \mathrm{mol} \cdot \mathrm{kg}^{-1}$ & $\kappa / \mathrm{S} \cdot \mathrm{m}^{-1}$ & $m / \mathrm{mol} \cdot \mathrm{kg}^{-1}$ & $\kappa / \mathrm{S} \cdot \mathrm{m}^{-1}$ & $\mathrm{~m} / \mathrm{mol} \cdot \mathrm{kg}^{-1}$ & $\overline{\kappa / S} \cdot \mathrm{m}^{-1}$ \\
\hline 0.13379 & 0.6691 & 0.13976 & 0.8091 & 0.10796 & 0.8218 & 0.13463 & 1.0416 \\
\hline 0.13723 & 0.6771 & 0.14307 & 0.8184 & 0.11082 & 0.8328 & 0.13808 & 1.0554 \\
\hline 0.14061 & 0.6850 & 0.14632 & 0.8274 & 0.11362 & 0.8436 & 0.14147 & 1.0688 \\
\hline 0.14393 & 0.6927 & 0.14952 & 0.8363 & 0.11637 & 0.8538 & 0.14481 & 1.0820 \\
\hline 0.14719 & 0.7002 & 0.15266 & 0.8449 & 0.11907 & 0.8638 & 0.14808 & 1.0947 \\
\hline 0.15040 & 0.7073 & 0.15575 & 0.8533 & 0.12173 & 0.8735 & 0.15130 & 1.1072 \\
\hline 0.15355 & 0.7141 & 0.15879 & 0.8615 & 0.12433 & 0.8828 & 0.15446 & 1.1192 \\
\hline 0.15665 & 0.7210 & 0.16177 & 0.8695 & 0.12689 & 0.8918 & 0.15757 & 1.1309 \\
\hline 0.15970 & 0.7276 & 0.16471 & 0.8773 & 0.12940 & 0.9008 & & \\
\hline 0.16269 & 0.7340 & 0.16760 & 0.8849 & 0.13188 & 0.9093 & & \\
\hline 0.16564 & 0.7403 & 0.17045 & 0.8922 & 0.13430 & 0.9175 & & \\
\hline 0.16854 & 0.7463 & 0.17325 & 0.8992 & 0.13669 & 0.9256 & & \\
\hline 0.17140 & 0.7525 & 0.17600 & 0.9061 & 0.13904 & 0.9336 & & \\
\hline 0.17420 & 0.7591 & 0.17871 & 0.9126 & 0.14134 & 0.9415 & & \\
\hline 0.17697 & 0.7642 & 0.18138 & 0.9188 & 0.14361 & 0.9490 & & \\
\hline 0.17968 & 0.7700 & 0.18401 & 0.9248 & 0.14584 & 0.9562 & & \\
\hline 0.18236 & 0.7756 & 0.18660 & 0.9304 & 0.14804 & 0.9635 & & \\
\hline 0.18500 & 0.7814 & 0.18915 & 0.9357 & 0.15020 & 0.9703 & & \\
\hline 0.18759 & 0.7863 & 0.19166 & 0.9407 & 0.15232 & 0.9771 & & \\
\hline 0.19014 & 0.7914 & 0.19413 & 0.9453 & 0.15441 & 0.9840 & & \\
\hline 0.19266 & 0.7964 & 0.19657 & 0.9495 & 0.15647 & 0.9904 & & \\
\hline 0.19514 & 0.8012 & & & 0.15850 & 0.9971 & & \\
\hline 0.19758 & 0.8062 & & & 0.16049 & 1.0032 & & \\
\hline 0.19998 & 0.8108 & & & 0.16245 & 1.0094 & & \\
\hline 0.20235 & 0.8149 & & & 0.16438 & 1.0158 & & \\
\hline 0.20469 & 0.8192 & & & 0.166287 & 1.0217 & & \\
\hline 0.20699 & 0.8233 & & & 0.16816 & 1.0275 & & \\
\hline 0.20925 & 0.8272 & & & 0.17001 & 1.0329 & & \\
\hline 0.21149 & 0.8311 & & & 0.17183 & 1.0381 & & \\
\hline 0.21369 & 0.8349 & & & 0.17362 & 1.0433 & & \\
\hline 0.21586 & 0.8384 & & & 0.17538 & 1.0487 & & \\
\hline 0.21801 & 0.8418 & & & 0.177123 & 1.0539 & & \\
\hline
\end{tabular}

Standard uncertainties are: $u(\mathrm{~T})=0.1 K, u(\mathrm{~m})=10^{-5} \mathrm{~m}, u(p)=0.05 p, u(\mathrm{wt} \%)=10^{-3} w t^{0} \%$ and the combined expanded uncertainty $U_{c}(\kappa)=0.004 \kappa($ level of confidence $=0.95)$. 
Table S4. Values of conductivity $\kappa$ for different concentration $-m$ of aqueous solutions of 8-8-8 surfactants within $288.15-303.15 \mathrm{~K}$ temperature range $(p=0.1 \mathrm{MPa})$.

\begin{tabular}{|c|c|c|c|c|c|c|c|}
\hline \multicolumn{2}{|c|}{$288.15 \mathrm{~K}$} & \multicolumn{2}{|c|}{$293.15 \mathrm{~K}$} & \multicolumn{2}{|c|}{$298.15 \mathrm{~K}$} & \multicolumn{2}{|c|}{$303.15 \mathrm{~K}$} \\
\hline$m / \mathrm{mol} \cdot \mathrm{kg}^{-1}$ & $\kappa / \mathrm{S} \cdot \mathrm{m}^{-1}$ & $\mathrm{~m} / \mathrm{mol} \cdot \mathrm{kg}^{-1}$ & $\kappa / S \cdot \mathrm{m}^{-1}$ & $m / \mathrm{mol} \cdot \mathrm{kg}^{-1}$ & $\kappa / S \cdot \mathrm{m}^{-1}$ & $m / \mathrm{mol}^{\prime} \mathrm{kg}^{-1}$ & $\kappa / \mathrm{S} \cdot \mathrm{m}^{-1}$ \\
\hline 0.00336 & 0.0505 & 0.00410 & 0.0582 & 0.00320 & 0.0429 & 0.00365 & 0.0690 \\
\hline 0.00663 & 0.0932 & 0.00810 & 0.1144 & 0.00631 & 0.0986 & 0.00720 & 0.1390 \\
\hline 0.00981 & 0.1321 & 0.01200 & 0.1652 & 0.00934 & 0.1476 & 0.01065 & 0.2009 \\
\hline 0.01290 & 0.1683 & 0.01579 & 0.2119 & 0.01230 & 0.1943 & 0.01401 & 0.2586 \\
\hline 0.01592 & 0.2016 & 0.01949 & 0.2553 & 0.01519 & 0.2376 & 0.01728 & 0.3110 \\
\hline 0.01886 & 0.2324 & 0.02310 & 0.2955 & 0.01800 & 0.2782 & 0.02047 & 0.3605 \\
\hline 0.02172 & 0.2617 & 0.02663 & 0.3335 & 0.02074 & 0.3165 & 0.02357 & 0.4071 \\
\hline 0.02451 & 0.2891 & 0.03006 & 0.3691 & 0.02342 & 0.3520 & 0.02660 & 0.4503 \\
\hline 0.02723 & 0.3153 & 0.03342 & 0.4026 & 0.02604 & 0.3857 & 0.02955 & 0.4905 \\
\hline 0.02989 & 0.3399 & 0.03669 & 0.4344 & 0.02859 & 0.4174 & 0.03243 & 0.5267 \\
\hline 0.03248 & 0.3632 & 0.03989 & 0.4642 & 0.03109 & 0.4477 & 0.03523 & 0.5629 \\
\hline 0.03501 & 0.3851 & 0.04301 & 0.4923 & 0.03353 & 0.4764 & 0.03797 & 0.5998 \\
\hline 0.03748 & 0.4064 & 0.04607 & 0.5188 & 0.03591 & 0.5034 & 0.04065 & 0.6325 \\
\hline 0.03989 & 0.4265 & 0.04905 & 0.5437 & 0.03824 & 0.5292 & 0.04326 & 0.6617 \\
\hline 0.04225 & 0.4454 & 0.05197 & 0.5670 & 0.04052 & 0.5538 & 0.04581 & 0.6911 \\
\hline 0.04455 & 0.4635 & 0.05483 & 0.5887 & 0.04275 & 0.5774 & 0.04830 & 0.7168 \\
\hline 0.04680 & 0.4814 & 0.05762 & 0.6090 & 0.04493 & 0.5998 & 0.05074 & 0.7398 \\
\hline 0.04900 & 0.4962 & 0.06035 & 0.6279 & 0.04706 & 0.6208 & 0.05312 & 0.7667 \\
\hline 0.05116 & 0.5107 & 0.06302 & 0.6451 & 0.04915 & 0.6405 & 0.05545 & 0.7853 \\
\hline 0.05326 & 0.5240 & 0.06564 & 0.6601 & 0.05119 & 0.6584 & 0.05772 & 0.8097 \\
\hline 0.05532 & 0.5388 & 0.06820 & 0.6739 & 0.05319 & 0.6728 & 0.05995 & 0.8284 \\
\hline 0.05734 & 0.5518 & 0.07071 & 0.6880 & 0.05515 & 0.6898 & 0.06213 & 0.8443 \\
\hline 0.05931 & 0.5643 & 0.07317 & 0.7011 & 0.05708 & 0.7062 & 0.06426 & 0.8636 \\
\hline 0.06124 & 0.5764 & 0.07558 & 0.7134 & 0.05896 & 0.7214 & 0.06635 & 0.8772 \\
\hline 0.06313 & 0.5875 & 0.07794 & 0.7250 & 0.06080 & 0.7358 & 0.06840 & 0.8926 \\
\hline 0.06499 & 0.5979 & 0.08025 & 0.7360 & 0.06261 & 0.7495 & 0.07040 & 0.9064 \\
\hline 0.06680 & 0.6082 & 0.08252 & 0.7465 & 0.06438 & 0.7621 & 0.07236 & 0.9199 \\
\hline 0.06858 & 0.6183 & 0.08475 & 0.7565 & 0.06612 & 0.7741 & 0.07428 & 0.9326 \\
\hline 0.07032 & 0.6257 & 0.08693 & 0.7660 & 0.06783 & 0.7855 & 0.07617 & 0.9448 \\
\hline 0.07203 & 0.6333 & 0.08907 & 0.7751 & 0.06950 & 0.7965 & 0.07801 & 0.9564 \\
\hline 0.07371 & 0.6407 & 0.09117 & 0.7838 & 0.07114 & 0.8065 & 0.07982 & 0.9651 \\
\hline 0.07535 & 0.6476 & 0.09323 & 0.7920 & 0.07275 & 0.8160 & 0.08160 & 0.9781 \\
\hline 0.07697 & 0.6543 & 0.09525 & 0.7999 & 0.07434 & 0.8253 & 0.08334 & 0.9891 \\
\hline 0.07855 & 0.6612 & 0.09723 & 0.8076 & 0.07589 & 0.8343 & 0.08504 & 0.9982 \\
\hline 0.08010 & 0.6669 & 0.09918 & 0.8150 & 0.07741 & 0.8427 & 0.08672 & 1.0053 \\
\hline 0.08162 & 0.6729 & 0.10109 & 0.8220 & 0.07891 & 0.8510 & 0.08836 & 1.0170 \\
\hline
\end{tabular}


Table S4 continuation. Values of conductivity $\kappa$ for different concentration $-m$ of aqueous solutions of 8-8-8 surfactants within 288.15 - $303.15 \mathrm{~K}$ temperature range $(p=0.1 \mathrm{MPa})$.

\begin{tabular}{cccccccc}
\hline \multicolumn{2}{c}{$288.15 \mathrm{~K}$} & \multicolumn{2}{c}{$293.15 \mathrm{~K}$} & \multicolumn{2}{c}{$298.15 \mathrm{~K}$} & \multicolumn{2}{c}{$303.15 \mathrm{~K}$} \\
\hline$m / \mathrm{mol} \cdot \mathrm{kg}^{-1}$ & $\kappa / \mathrm{S} \cdot \mathrm{m}^{-1}$ & $m / \mathrm{mol} \cdot \mathrm{kg}^{-1}$ & $\kappa / \mathrm{S} \cdot \mathrm{m}^{-1}$ & $m / \mathrm{mol}^{-1} \mathrm{~kg}^{-1}$ & $\kappa / \mathrm{S} \cdot \mathrm{m}^{-1}$ & $m / \mathrm{mol}^{\prime} \mathrm{kg}^{-1}$ & $\kappa / \mathrm{S} \cdot \mathrm{m}^{-1}$ \\
\hline 0.08312 & 0.6782 & 0.10297 & 0.8288 & 0.08038 & 0.8585 & 0.08998 & 1.0271 \\
0.08459 & 0.6836 & 0.10482 & 0.8355 & 0.08183 & 0.8659 & 0.09156 & 1.0347 \\
0.08603 & 0.6883 & 0.10663 & 0.8410 & 0.08324 & 0.8731 & 0.09312 & 1.0443 \\
0.08745 & 0.6929 & 0.10841 & 0.8473 & 0.08464 & 0.8795 & 0.09464 & 1.0515 \\
0.08884 & 0.6978 & 0.11016 & 0.8534 & 0.08601 & 0.8852 & 0.09614 & 1.0583 \\
0.09020 & 0.7032 & 0.11188 & 0.8595 & 0.08736 & 0.8918 & 0.09762 & 1.0675 \\
0.09155 & 0.7075 & 0.11357 & 0.8655 & 0.08868 & 0.8983 & 0.09906 & 1.0745 \\
0.09287 & 0.7107 & 0.11524 & 0.8711 & 0.08998 & 0.9052 & 0.10049 & 1.0827 \\
0.09416 & 0.7171 & 0.11687 & 0.8766 & 0.09126 & 0.9113 & 0.10188 & 1.0887 \\
0.09544 & 0.7187 & 0.11848 & 0.8820 & 0.09252 & 0.9172 & 0.10326 & 1.0970 \\
0.09669 & 0.7229 & 0.12006 & 0.8873 & 0.09376 & 0.9233 & 0.10461 & 1.1025 \\
0.09792 & 0.7267 & 0.12162 & 0.8924 & 0.09498 & 0.9287 & 0.10594 & 1.1025 \\
0.09913 & 0.7304 & 0.12315 & 0.8974 & 0.09618 & 0.9342 & 0.10724 & 1.1159 \\
0.10033 & 0.7341 & 0.12466 & 0.9022 & 0.09736 & 0.9389 & 0.10853 & 1.1225 \\
\hline
\end{tabular}

Standard uncertainties are: $u(\mathrm{~T})=0.1 K, u(\mathrm{~m})=10^{-5} \mathrm{~m}, u(p)=0.05 p$ and the combined expanded uncertainty $U_{c}(\kappa)=0.004 \kappa($ level of confidence $=0.95)$.

Table S5. Values of conductivity $\kappa$ for different concentration $-m$ of aqueous solutions of 8-8-8 surfactants within 308.15 - 323.15 K temperature range $(p=0.1 \mathrm{MPa})$.

\begin{tabular}{cccccccc}
\hline \multicolumn{2}{c}{$308.15 \mathrm{~K}$} & \multicolumn{2}{c}{$313.15 \mathrm{~K}$} & \multicolumn{2}{c}{$318.15 \mathrm{~K}$} & \multicolumn{2}{c}{$323.15 \mathrm{~K}$} \\
\hline $\mathrm{m} / \mathrm{mol} \cdot \mathrm{kg}^{-1}$ & $\kappa / \mathrm{S} \cdot \mathrm{m}^{-1}$ & $\mathrm{~m} / \mathrm{mol} \cdot \mathrm{kg}^{-1}$ & $\kappa / \mathrm{S} \cdot \mathrm{m}^{-1}$ & $\mathrm{~m} / \mathrm{mol} \cdot \mathrm{kg}^{-1}$ & $\kappa / \mathrm{S} \cdot \mathrm{m}^{-1}$ & $\mathrm{~m} / \mathrm{mol} \cdot \mathrm{kg}^{-1}$ & $\kappa / \mathrm{S} \cdot \mathrm{m}^{-1}$ \\
\hline 0.00373 & 0.0893 & 0.00397 & 0.1011 & 0.00377 & 0.1083 & 0.00376 & 0.1151 \\
0.00736 & 0.1665 & 0.00784 & 0.1891 & 0.00744 & 0.2002 & 0.00741 & 0.2165 \\
0.01089 & 0.2354 & 0.01160 & 0.2694 & 0.01101 & 0.2877 & 0.01096 & 0.3071 \\
0.01432 & 0.2994 & 0.01526 & 0.3431 & 0.01448 & 0.3660 & 0.01442 & 0.3918 \\
0.01766 & 0.3593 & 0.01882 & 0.4106 & 0.01786 & 0.4391 & 0.01779 & 0.4688 \\
0.02091 & 0.4137 & 0.02229 & 0.4749 & 0.02115 & 0.5069 & 0.02106 & 0.5416 \\
0.02407 & 0.4656 & 0.02567 & 0.5346 & 0.02435 & 0.5705 & 0.02425 & 0.6105 \\
0.02716 & 0.5140 & 0.02897 & 0.5903 & 0.02747 & 0.6295 & 0.02736 & 0.6732 \\
0.03016 & 0.5602 & 0.03218 & 0.6421 & 0.03051 & 0.6854 & 0.03040 & 0.7335 \\
0.03309 & 0.6034 & 0.03532 & 0.6919 & 0.03348 & 0.7376 & 0.03335 & 0.7881 \\
0.03594 & 0.6441 & 0.03837 & 0.7377 & 0.03637 & 0.7865 & 0.03623 & 0.8389 \\
0.03873 & 0.6822 & 0.04136 & 0.7807 & 0.03919 & 0.8321 & 0.03905 & 0.8888 \\
0.04145 & 0.7178 & 0.04427 & 0.8213 & 0.04194 & 0.8747 & 0.04179 & 0.9349 \\
0.04410 & 0.7517 & 0.04712 & 0.8594 & 0.04463 & 0.9153 & 0.04447 & 0.9796 \\
\hline
\end{tabular}


Table S5 continuation. Values of conductivity $\kappa$ for different concentration $-m$ of aqueous solutions of 8-8-8 surfactants within 308.15 - 323.15 $\mathrm{K}$ temperature range $(p=$ 0.1 MPa).

\begin{tabular}{|c|c|c|c|c|c|c|c|}
\hline \multicolumn{2}{|c|}{$308.15 \mathrm{~K}$} & \multicolumn{2}{|c|}{$313.15 \mathrm{~K}$} & \multicolumn{2}{|c|}{$318.15 \mathrm{~K}$} & \multicolumn{2}{|c|}{$323.15 \mathrm{~K}$} \\
\hline $\mathrm{m} / \mathrm{mol} \cdot \mathrm{kg}^{-1}$ & $\kappa / \mathrm{S} \cdot \mathrm{m}^{-1}$ & $m / \mathrm{mol} \cdot \mathrm{kg}^{-1}$ & $\kappa / \mathrm{S} \cdot \mathrm{m}^{-1}$ & $m / \mathrm{mol} \cdot \mathrm{kg}^{-1}$ & $\kappa / \mathrm{S} \cdot \mathrm{m}^{-1}$ & $m / \mathrm{mol} \cdot \mathrm{kg}^{-1}$ & $\kappa / \mathrm{S} \cdot \mathrm{m}^{-1}$ \\
\hline 0.04669 & 0.7836 & 0.04990 & 0.8944 & 0.04725 & 0.9537 & 0.04709 & 1.0187 \\
\hline 0.04922 & 0.8135 & 0.05261 & 0.9280 & 0.04981 & 0.9894 & 0.04964 & 1.0578 \\
\hline 0.05169 & 0.8412 & 0.05526 & 0.9585 & 0.05232 & 1.0236 & 0.05214 & 1.0944 \\
\hline 0.05411 & 0.8667 & 0.05786 & 0.9872 & 0.05476 & 1.0545 & 0.05458 & 1.1301 \\
\hline 0.05647 & 0.8904 & 0.06039 & 1.0137 & 0.05716 & 1.0830 & 0.05696 & 1.1603 \\
\hline 0.05877 & 0.9135 & 0.06287 & 1.0387 & 0.05949 & 1.1106 & 0.05930 & 1.1905 \\
\hline 0.06103 & 0.9341 & 0.06530 & 1.0626 & 0.06178 & 1.1355 & 0.06158 & 1.2211 \\
\hline 0.06323 & 0.9538 & 0.06768 & 1.0846 & 0.06402 & 1.1598 & 0.06381 & 1.2492 \\
\hline 0.06539 & 0.9725 & 0.07000 & 1.1059 & 0.06620 & 1.1827 & 0.06599 & 1.2749 \\
\hline 0.06750 & 0.9895 & 0.07227 & 1.1257 & 0.06835 & 1.2042 & 0.06813 & 1.2995 \\
\hline 0.06957 & 1.0066 & 0.07450 & 1.1449 & 0.07044 & 1.2255 & 0.07022 & 1.3225 \\
\hline 0.07160 & 1.0218 & 0.07668 & 1.1626 & 0.07250 & 1.2469 & 0.07227 & 1.3453 \\
\hline 0.07358 & 1.0368 & 0.07882 & 1.1802 & 0.07451 & 1.2657 & 0.07428 & 1.3658 \\
\hline 0.07552 & 1.0512 & 0.08091 & 1.1970 & 0.07648 & 1.2856 & 0.07624 & 1.3873 \\
\hline 0.07742 & 1.0645 & 0.08297 & 1.2125 & 0.07840 & 1.3021 & 0.07817 & 1.4084 \\
\hline 0.07929 & 1.0778 & 0.08498 & 1.2276 & 0.08030 & 1.3188 & 0.08005 & 1.4260 \\
\hline 0.08111 & 1.0898 & 0.08695 & 1.2423 & 0.08215 & 1.3355 & 0.08190 & 1.4441 \\
\hline 0.08290 & 1.1017 & 0.08888 & 1.2558 & 0.08396 & 1.3498 & 0.08372 & 1.4621 \\
\hline 0.08466 & 1.1137 & 0.09078 & 1.2703 & 0.08575 & 1.3640 & 0.08550 & 1.4773 \\
\hline 0.08638 & 1.1248 & 0.09264 & 1.2816 & 0.08749 & 1.3798 & 0.08724 & 1.4945 \\
\hline 0.08807 & 1.1353 & 0.09447 & 1.2966 & 0.08921 & 1.3949 & 0.08895 & 1.5098 \\
\hline 0.08973 & 1.1461 & 0.09626 & 1.3080 & 0.09089 & 1.4077 & 0.09063 & 1.5248 \\
\hline 0.09135 & 1.1562 & 0.09802 & 1.3205 & 0.09254 & 1.4215 & 0.09228 & 1.5399 \\
\hline 0.09451 & 1.1745 & 0.10144 & 1.3426 & 0.09575 & 1.4452 & 0.09548 & 1.5665 \\
\hline 0.09605 & 1.1845 & 0.10310 & 1.3531 & 0.09731 & 1.4588 & 0.09704 & 1.5786 \\
\hline 0.09756 & 1.1931 & 0.10473 & 1.3640 & 0.09884 & 1.4675 & 0.09857 & 1.5922 \\
\hline 0.09904 & 1.2015 & 0.10634 & 1.3731 & 0.10034 & 1.4782 & 0.10008 & 1.6049 \\
\hline 0.10050 & 1.2098 & 0.10792 & 1.3848 & 0.10182 & 1.4888 & 0.10155 & 1.6169 \\
\hline 0.10193 & 1.2178 & 0.10947 & 1.3940 & 0.10327 & 1.4988 & 0.10300 & 1.6308 \\
\hline 0.10333 & 1.2266 & 0.11099 & 1.4031 & 0.10470 & 1.5091 & 0.10443 & 1.6419 \\
\hline 0.10471 & 1.2339 & 0.11249 & 1.4143 & 0.10610 & 1.5194 & 0.10583 & 1.6544 \\
\hline 0.10607 & 1.2421 & 0.11396 & 1.4228 & 0.10748 & 1.5296 & 0.10721 & 1.6646 \\
\hline 0.10740 & 1.2488 & 0.11541 & 1.4312 & 0.10884 & 1.5406 & 0.10856 & 1.6758 \\
\hline 0.10871 & 1.2565 & 0.11683 & 1.4396 & 0.11017 & 1.5495 & 0.10989 & 1.6870 \\
\hline 0.11000 & 1.2646 & 0.11823 & 1.4484 & 0.11148 & 1.5592 & 0.11120 & 1.6973 \\
\hline
\end{tabular}

Standard uncertainties are: $u(\mathrm{~T})=0.1 K, u(\mathrm{~m})=10^{-5} m, u(p)=0.05 p$ and the combined expanded uncertainty $U_{c}(\kappa)=0.004 \kappa($ level of confidence $=0.95)$. 
Table S6. Values of conductivity $\kappa$ for different concentration $-m$ of 8-8-8 surfactant in $10 \mathrm{wt} \%$ ethanol-water mixtures of within 288.15 - 303.15 K temperature range $(p=$

\subsection{MPa).}

\begin{tabular}{|c|c|c|c|c|c|c|c|}
\hline \multicolumn{2}{|c|}{$288.15 \mathrm{~K}$} & \multicolumn{2}{|c|}{$293.15 \mathrm{~K}$} & \multicolumn{2}{|c|}{$298.15 \mathrm{~K}$} & \multicolumn{2}{|c|}{$303.15 \mathrm{~K}$} \\
\hline$m / \mathrm{mol} \cdot \mathrm{kg}^{-1}$ & $\kappa / \mathrm{S} \cdot \mathrm{m}^{-1}$ & $m / \mathrm{mol} \cdot \mathrm{kg}^{-1}$ & $\kappa / \mathrm{S} \cdot \mathrm{m}^{-1}$ & $m / \mathrm{mol}^{\prime} \mathrm{kg}^{-1}$ & $\kappa / \mathrm{S} \cdot \mathrm{m}^{-1}$ & $\mathrm{~m} / \mathrm{mol} \cdot \mathrm{kg}^{-1}$ & $\mathrm{\kappa} / \mathrm{S} \cdot \mathrm{m}^{-1}$ \\
\hline 0.00567 & 0.0579 & 0.00563 & 0.0691 & 0.00480 & 0.0699 & 0.00477 & 0.0790 \\
\hline 0.00777 & 0.0772 & 0.00772 & 0.0903 & 0.00949 & 0.1230 & 0.00760 & 0.1156 \\
\hline 0.00986 & 0.0955 & 0.00979 & 0.1109 & 0.01406 & 0.1755 & 0.00943 & 0.1390 \\
\hline 0.01192 & 0.1129 & 0.01184 & 0.1309 & 0.01575 & 0.1939 & 0.01170 & 0.1677 \\
\hline 0.01397 & 0.1279 & 0.01387 & 0.1505 & 0.01742 & 0.2119 & 0.01397 & 0.1960 \\
\hline 0.01600 & 0.1458 & 0.01589 & 0.1695 & 0.01908 & 0.2290 & 0.01565 & 0.2166 \\
\hline 0.01801 & 0.1614 & 0.01789 & 0.1879 & 0.02072 & 0.2454 & 0.01731 & 0.2367 \\
\hline 0.02000 & 0.1763 & 0.01987 & 0.2058 & 0.02235 & 0.2615 & 0.01895 & 0.2563 \\
\hline 0.02198 & 0.1908 & 0.02183 & 0.2231 & 0.02396 & 0.2772 & 0.02059 & 0.2754 \\
\hline 0.02393 & 0.2048 & 0.02378 & 0.2398 & 0.02556 & 0.2925 & 0.02221 & 0.2940 \\
\hline 0.02588 & 0.2184 & 0.02570 & 0.2560 & 0.02714 & 0.3075 & 0.02381 & 0.3121 \\
\hline 0.02780 & 0.2315 & 0.02762 & 0.2717 & 0.02871 & 0.3219 & 0.02540 & 0.3297 \\
\hline 0.02971 & 0.2441 & 0.02951 & 0.2868 & 0.03027 & 0.3360 & 0.02697 & 0.3469 \\
\hline 0.03160 & 0.2564 & 0.03139 & 0.3014 & 0.03181 & 0.3498 & 0.02854 & 0.3635 \\
\hline 0.03347 & 0.2683 & 0.03325 & 0.3155 & 0.03334 & 0.3633 & 0.03008 & 0.3796 \\
\hline 0.03533 & 0.2797 & 0.03510 & 0.3291 & 0.03486 & 0.3764 & 0.03162 & 0.3953 \\
\hline 0.04018 & 0.3082 & 0.03992 & 0.3629 & 0.03636 & 0.3892 & 0.03314 & 0.4105 \\
\hline 0.04492 & 0.3343 & 0.04463 & 0.3936 & 0.03785 & 0.4015 & 0.03465 & 0.4253 \\
\hline 0.04956 & 0.3581 & 0.04925 & 0.4216 & 0.03933 & 0.4137 & 0.03614 & 0.4396 \\
\hline 0.05409 & 0.3797 & 0.05376 & 0.4472 & 0.04080 & 0.4256 & 0.03763 & 0.4535 \\
\hline 0.05853 & 0.3991 & 0.05817 & 0.4706 & 0.04225 & 0.4370 & 0.03910 & 0.4670 \\
\hline 0.06288 & 0.4167 & 0.06249 & 0.4921 & 0.04369 & 0.4482 & 0.04055 & 0.4801 \\
\hline 0.06713 & 0.4328 & 0.06673 & 0.5119 & 0.04512 & 0.4589 & 0.04200 & 0.4928 \\
\hline 0.07129 & 0.4477 & 0.07087 & 0.5303 & 0.04653 & 0.4694 & 0.04343 & 0.5051 \\
\hline 0.07537 & 0.4615 & 0.07493 & 0.5475 & 0.04794 & 0.4797 & 0.04485 & 0.5171 \\
\hline 0.07937 & 0.4744 & 0.07891 & 0.5636 & 0.04933 & 0.4898 & 0.04626 & 0.5287 \\
\hline 0.08329 & 0.4866 & 0.08280 & 0.5788 & 0.05071 & 0.4995 & 0.04766 & 0.5413 \\
\hline 0.08712 & 0.4978 & 0.08662 & 0.5931 & 0.05208 & 0.5086 & 0.04905 & 0.5595 \\
\hline 0.09088 & 0.5086 & 0.09037 & 0.6068 & 0.05568 & 0.5306 & 0.05042 & 0.5659 \\
\hline 0.09457 & 0.5188 & 0.09404 & 0.6198 & 0.05919 & 0.5486 & 0.05178 & 0.5754 \\
\hline 0.09819 & 0.5285 & 0.09764 & 0.6323 & 0.06264 & 0.5695 & 0.05536 & 0.5982 \\
\hline 0.10173 & 0.5376 & 0.10117 & 0.6443 & 0.06601 & 0.5868 & 0.05886 & 0.6226 \\
\hline 0.10521 & 0.5466 & 0.10464 & 0.6559 & 0.06931 & 0.6027 & 0.06229 & 0.6455 \\
\hline 0.10863 & 0.5553 & 0.10804 & 0.6670 & 0.07254 & 0.6204 & 0.06564 & 0.6670 \\
\hline 0.11198 & 0.5635 & 0.11138 & 0.6777 & 0.07570 & 0.6371 & 0.06893 & 0.6872 \\
\hline
\end{tabular}


Table S6 continuation. Values of conductivity $\kappa$ for different concentration $-m$ of 8-8-8 surfactant in $10 \mathrm{wt} \%$ ethanol-water mixtures within $288.15-303.15 \mathrm{~K}$ temperature range $(p=0.1 \mathrm{MPa})$.

\begin{tabular}{|c|c|c|c|c|c|c|c|}
\hline \multicolumn{2}{|c|}{$288.15 \mathrm{~K}$} & \multicolumn{2}{|c|}{$293.15 \mathrm{~K}$} & \multicolumn{2}{|c|}{$298.15 \mathrm{~K}$} & \multicolumn{2}{|c|}{$303.15 \mathrm{~K}$} \\
\hline$m / \mathrm{mol}^{\prime} \mathrm{kg}^{-1}$ & $\kappa / \mathrm{S} \cdot \mathrm{m}^{-1}$ & $m / \mathrm{mol} \cdot \mathrm{kg}^{-1}$ & $\kappa / \mathrm{S} \cdot \mathrm{m}^{-1}$ & $m / \mathrm{mol} \cdot \mathrm{kg}^{-1}$ & $\kappa / \mathrm{S} \cdot \mathrm{m}^{-1}$ & $m / \mathrm{mol}^{\prime} \mathrm{kg}^{-1}$ & $\kappa / \mathrm{S} \cdot \mathrm{m}^{-1}$ \\
\hline 0.11527 & 0.5715 & 0.11465 & 0.6881 & 0.07881 & 0.6529 & 0.07214 & 0.7063 \\
\hline 0.11849 & 0.5793 & 0.11787 & 0.6982 & 0.08185 & 0.6679 & 0.07530 & 0.7245 \\
\hline 0.12166 & 0.5866 & 0.12103 & 0.7079 & 0.08483 & 0.6821 & 0.07839 & 0.7419 \\
\hline 0.12477 & 0.5940 & 0.12413 & 0.7172 & 0.08775 & 0.6957 & 0.08142 & 0.7585 \\
\hline 0.12783 & 0.6010 & 0.12718 & 0.7263 & 0.09061 & 0.7090 & 0.08438 & 0.7745 \\
\hline 0.13083 & 0.6078 & 0.13017 & 0.7350 & 0.09342 & 0.7208 & 0.08730 & 0.7898 \\
\hline 0.13378 & 0.6144 & 0.13311 & 0.7435 & 0.09618 & 0.7325 & 0.09015 & 0.8046 \\
\hline 0.13668 & 0.6209 & 0.13600 & 0.7517 & 0.09888 & 0.7439 & 0.09295 & 0.8189 \\
\hline 0.13953 & 0.6272 & 0.13884 & 0.7596 & 0.10154 & 0.7547 & 0.09570 & 0.8328 \\
\hline 0.14233 & 0.6332 & 0.14163 & 0.7672 & 0.10414 & 0.7654 & 0.09839 & 0.8462 \\
\hline 0.14509 & 0.6390 & 0.14438 & 0.7746 & 0.10670 & 0.7755 & 0.10104 & 0.8592 \\
\hline 0.14779 & 0.6446 & 0.14708 & 0.7818 & 0.10921 & 0.7850 & 0.10364 & 0.8719 \\
\hline 0.15045 & 0.6492 & 0.14973 & 0.7888 & 0.11168 & 0.7946 & 0.10619 & 0.8842 \\
\hline 0.15307 & 0.6543 & 0.15235 & 0.7955 & 0.11410 & 0.8039 & 0.10869 & 0.8962 \\
\hline 0.15565 & 0.6592 & 0.15491 & 0.8022 & 0.11648 & 0.8097 & 0.11115 & 0.9078 \\
\hline 0.15818 & 0.6642 & 0.15744 & 0.8086 & 0.11882 & 0.8204 & 0.11357 & 0.9191 \\
\hline 0.16067 & 0.6687 & 0.15993 & 0.8150 & 0.12112 & 0.8300 & 0.11594 & 0.9300 \\
\hline 0.16312 & 0.6734 & 0.16237 & 0.8213 & 0.12337 & 0.8375 & 0.11827 & 0.9407 \\
\hline 0.16554 & 0.6779 & 0.16478 & 0.8276 & 0.12559 & 0.8461 & 0.12056 & 0.9510 \\
\hline 0.16791 & 0.6824 & 0.16715 & 0.8338 & 0.12778 & 0.8586 & 0.12282 & 0.9610 \\
\hline 0.17025 & 0.6866 & 0.16949 & 0.8401 & 0.12992 & 0.8615 & 0.12503 & 0.9707 \\
\hline 0.17255 & 0.6908 & 0.17179 & 0.8465 & 0.13203 & 0.8732 & 0.12721 & 0.9802 \\
\hline 0.17482 & 0.6950 & 0.17405 & 0.8530 & 0.13410 & 0.8760 & 0.12935 & 0.9893 \\
\hline 0.17705 & 0.6990 & 0.17628 & 0.8596 & 0.13615 & 0.8871 & 0.13145 & 0.9982 \\
\hline 0.17925 & 0.7027 & & & 0.13815 & 0.8898 & 0.13352 & 1.0068 \\
\hline 0.18142 & 0.7067 & & & 0.14013 & 0.9004 & 0.13556 & 1.0151 \\
\hline 0.18355 & 0.7105 & & & 0.14207 & 0.9027 & 0.13756 & 1.0232 \\
\hline 0.18565 & 0.7144 & & & 0.14398 & 0.9130 & 0.13953 & 1.0311 \\
\hline 0.18772 & 0.7182 & & & 0.14587 & 0.9152 & 0.14147 & 1.0387 \\
\hline 0.18976 & 0.7216 & & & 0.14772 & 0.9250 & 0.14338 & 1.0462 \\
\hline 0.19177 & 0.7251 & & & 0.14955 & 0.9270 & 0.14526 & 1.0534 \\
\hline \multirow[t]{4}{*}{0.19376} & 0.7285 & & & 0.15134 & 0.9365 & 0.14711 & 1.0605 \\
\hline & & & & 0.15311 & 0.9385 & 0.14894 & 1.0674 \\
\hline & & & & 0.15485 & 0.9474 & 0.15073 & 1.0742 \\
\hline & & & & 0.15657 & 0.9497 & 0.15250 & 1.0809 \\
\hline
\end{tabular}


Table S6 continuation. Values of conductivity $\kappa$ for different concentration $-m$ of 8-8-8 surfactant in $10 \mathrm{wt} \%$ ethanol-water mixtures of within $288.15-303.15 \mathrm{~K}$ temperature range $(p=0.1 \mathrm{MPa})$.

\begin{tabular}{|c|c|c|c|c|c|c|c|}
\hline \multicolumn{2}{|c|}{$288.15 \mathrm{~K}$} & \multicolumn{2}{|c|}{$293.15 \mathrm{~K}$} & \multicolumn{2}{|c|}{$298.15 \mathrm{~K}$} & \multicolumn{2}{|c|}{$303.15 \mathrm{~K}$} \\
\hline $\mathrm{m} / \mathrm{mol} \cdot \mathrm{kg}^{-1}$ & $\kappa / \mathrm{S} \cdot \mathrm{m}^{-1}$ & $m / \mathrm{mol} \cdot \mathrm{kg}^{-1}$ & $\kappa / \mathrm{S} \cdot \mathrm{m}^{-1}$ & $m / \mathrm{mol} \cdot \mathrm{kg}^{-1}$ & $\kappa / \mathrm{S} \cdot \mathrm{m}^{-1}$ & $m / \mathrm{mol} \cdot \mathrm{kg}^{-1}$ & $\kappa / \mathrm{S} \cdot \mathrm{m}^{-1}$ \\
\hline & & & & 0.15826 & 0.9579 & 0.15424 & 1.0875 \\
\hline & & & & 0.15993 & 0.9629 & 0.15595 & 1.0940 \\
\hline & & & & 0.16157 & 0.9679 & 0.15764 & 1.1004 \\
\hline & & & & 0.16319 & 0.9727 & 0.15930 & 1.1069 \\
\hline & & & & 0.16478 & 0.9774 & 0.16094 & 1.1133 \\
\hline & & & & & & 0.16256 & 1.1198 \\
\hline & & & & & & 0.16415 & 1.1263 \\
\hline
\end{tabular}

Standard uncertainties are: $u(\mathrm{~T})=0.1 K, u(\mathrm{~m})=10^{-5} \mathrm{~m}, u(p)=0.20 \mathrm{KPa}, u(\mathrm{wt} \%)=10^{-3} \mathrm{wt} \%$ and the combined expanded uncertainty $U_{c}(\kappa)=0.004 \kappa($ level of confidence $=0.95)$.

Table S7. Values of surface tension $\gamma$ for different concentration $-m$ of aqueous and 10 wt\% ethanol solutions of 8-7-8 and 8-8-8 surfactants at 293.15 K $(p=0.1 \mathrm{MPa})$.

\begin{tabular}{|c|c|c|c|c|c|c|c|}
\hline \multicolumn{2}{|c|}{$8-7-8$} & \multicolumn{2}{|c|}{$8-7-8+10 w t \%$ EtOH } & \multicolumn{2}{|c|}{$8-8-8$} & \multicolumn{2}{|c|}{$8-8-8+10 w t \%$ EtOH } \\
\hline$m / \mathrm{mol}^{\prime} \cdot \mathrm{kg}^{-1}$ & $\gamma / \mathrm{mN} \cdot \mathrm{m}^{-1}$ & $m / \mathrm{mol} \cdot \mathrm{kg}^{-1}$ & $\gamma / \mathrm{mN} \cdot \mathrm{m}^{-1}$ & $m / \mathrm{mol} \cdot \mathrm{kg}^{-1}$ & $\gamma / \mathrm{mN} \cdot \mathrm{m}^{-1}$ & $m / \mathrm{mol} \cdot \mathrm{kg}^{-1}$ & $\gamma / \mathrm{mN} \cdot \mathrm{m}^{-1}$ \\
\hline 0.00000 & 72.8 & 0.00000 & 42.5 & 0.00000 & 72.8 & 0.00000 & 44.3 \\
\hline 0.00674 & 60.2 & 0.00386 & 40.9 & 0.00309 & 61.3 & 0.00471 & 41.0 \\
\hline 0.01312 & 52.6 & 0.00759 & 39.6 & 0.00613 & 55.1 & 0.00927 & 39.4 \\
\hline 0.01918 & 48.1 & 0.01120 & 38.5 & 0.00913 & 51.5 & 0.01367 & 38.4 \\
\hline 0.02493 & 45.8 & 0.01468 & 37.4 & 0.01207 & 49.0 & 0.01793 & 37.5 \\
\hline 0.03041 & 44.2 & 0.01806 & 36.2 & 0.01498 & 47.3 & 0.02206 & 36.7 \\
\hline 0.03562 & 43.3 & 0.02132 & 35.4 & 0.01783 & 45.9 & 0.02606 & 36.0 \\
\hline 0.04059 & 42.2 & 0.02449 & 34.8 & 0.02065 & 44.5 & 0.02993 & 35.3 \\
\hline 0.04533 & 41.4 & 0.02755 & 34.2 & 0.02341 & 43.1 & 0.03369 & 34.6 \\
\hline 0.04987 & 40.4 & 0.03053 & 33.8 & 0.02614 & 42.5 & 0.03734 & 34.2 \\
\hline 0.05420 & 39.5 & 0.03341 & 33.4 & 0.02883 & 41.6 & 0.04088 & 33.7 \\
\hline 0.05836 & 39.1 & 0.03621 & 33.0 & 0.03148 & 41.0 & 0.04431 & 33.4 \\
\hline 0.06233 & 38.4 & 0.03893 & 32.6 & 0.03408 & 40.3 & 0.04765 & 33.1 \\
\hline 0.06615 & 38.1 & 0.04157 & 32.3 & 0.03665 & 39.4 & 0.05089 & 32.8 \\
\hline 0.06981 & 37.8 & 0.04414 & 32.1 & 0.03919 & 39.1 & 0.05405 & 32.6 \\
\hline 0.07333 & 37.5 & 0.04663 & 31.9 & 0.04168 & 38.8 & 0.05711 & 32.5 \\
\hline 0.07672 & 37.4 & 0.04906 & 31.7 & 0.04414 & 38.6 & 0.06010 & 32.4 \\
\hline 0.07997 & 37.2 & 0.05142 & 31.6 & 0.04657 & 38.1 & 0.06300 & 32.3 \\
\hline 0.08311 & 37.3 & 0.05372 & 31.4 & 0.04896 & 37.9 & 0.06583 & 32.2 \\
\hline 0.08613 & 37.3 & 0.05595 & 31.3 & 0.05131 & 37.6 & 0.06858 & 32.1 \\
\hline 0.08905 & 37.4 & 0.05813 & 31.2 & 0.05364 & 37.4 & 0.07127 & 32.1 \\
\hline 0.09186 & 37.3 & 0.06026 & 31.1 & 0.05593 & 37.2 & 0.07388 & 32.1 \\
\hline 0.09457 & 37.4 & 0.06233 & 31.0 & 0.05819 & 37.3 & 0.07643 & 32.1 \\
\hline 0.09719 & 37.2 & 0.06434 & 31.0 & 0.06042 & 37.1 & 0.07892 & 32.1 \\
\hline 0.09973 & 37.1 & 0.06631 & 30.9 & 0.06262 & 37.0 & 0.08135 & 32.0 \\
\hline 0.10218 & 37.0 & 0.06823 & 30.8 & 0.06479 & 36.9 & 0.08372 & 32.0 \\
\hline 0.10456 & 37.0 & 0.07010 & 30.7 & 0.06693 & 37.0 & 0.08603 & 32.0 \\
\hline 0.10685 & 37.0 & 0.07193 & 30.7 & 0.06904 & 36.8 & 0.08829 & 31.9 \\
\hline
\end{tabular}


Table S7 continuation. Values of surface tension $\gamma$ for different concentration $-m$ of aqueous and $10 \mathrm{wt} \%$ ethanol solutions of 8-7-8 and 8-8-8 surfactants at 293.15 K ( $p=$ 0.1 MPa).

\begin{tabular}{|c|c|c|c|c|c|c|c|c|}
\hline \multicolumn{2}{|c|}{$8-7-8$} & \multicolumn{2}{|c|}{$8-7-8+10 \mathrm{wt} \%$ EtOH } & \multicolumn{3}{|c|}{$8-8-8$} & \multicolumn{2}{|c|}{$8-8-8+10 \mathrm{wt} \%$ EtOH } \\
\hline $\mathrm{m} / \mathrm{mol} \cdot \mathrm{kg}^{-1}$ & $\gamma / \mathrm{mN} \cdot \mathrm{m}^{-1}$ & $m / \mathrm{mol} \cdot \mathrm{kg}^{-1}$ & $\gamma / \mathrm{mN} \cdot \mathrm{m}^{-1}$ & $\mathrm{~m} / \mathrm{mol}$ & $\mathrm{kg}^{-1}$ & $\gamma / \mathrm{mN} \cdot \mathrm{m}^{-1}$ & $m / \mathrm{mol} \cdot \mathrm{kg}^{-1}$ & $\gamma / \mathrm{mN} \cdot \mathrm{m}^{-1}$ \\
\hline 0.10908 & 36.9 & 0.07372 & 30.6 & 0.07113 & & 36.7 & 0.09049 & 31.8 \\
\hline 0.11124 & 37.0 & 0.07546 & 30.5 & 0.07724 & & 36.6 & 0.09265 & 32.0 \\
\hline 0.11333 & 36.9 & 0.07717 & 30.5 & 0.08312 & & 36.9 & 0.09475 & 32.0 \\
\hline 0.11536 & 36.9 & 0.07883 & 30.4 & 0.08878 & & 36.7 & 0.09681 & 31.9 \\
\hline & & 0.08046 & 30.4 & 0.09423 & & 36.9 & 0.09882 & 31.9 \\
\hline & & 0.08205 & 30.3 & 0.09948 & & 36.6 & 0.10079 & 31.8 \\
\hline & & 0.08361 & 30.2 & 0.10455 & & 36.9 & 0.10272 & 32.0 \\
\hline & & 0.08513 & 30.2 & 0.10944 & & 36.4 & 0.10460 & 32.0 \\
\hline & & 0.08662 & 30.2 & 0.11416 & & 36.6 & 0.10644 & 31.9 \\
\hline & & 0.08808 & 30.2 & 0.11872 & & 36.4 & 0.10825 & 32.0 \\
\hline & & 0.08950 & 30.2 & 0.12313 & & 36.5 & 0.11002 & 32.0 \\
\hline & & 0.09090 & 30.1 & & & & 0.11175 & 31.8 \\
\hline & & 0.09227 & 30.1 & & & & 0.11344 & 31.9 \\
\hline & & 0.09361 & 30.2 & & & & 0.11510 & 32.0 \\
\hline & & 0.09492 & 30.2 & & & & 0.11673 & 32.0 \\
\hline & & 0.09621 & 30.1 & & & & 0.11833 & 31.8 \\
\hline & & 0.09747 & 30.1 & & & & 0.11989 & 32.0 \\
\hline & & 0.09871 & 30.0 & & & & 0.12143 & 32.1 \\
\hline & & 0.09992 & 30.1 & & & & 0.12293 & 32.0 \\
\hline & & 0.10111 & 30.0 & & & & 0.12441 & 32.0 \\
\hline & & 0.10228 & 30.1 & & & & 0.12586 & 31.8 \\
\hline & & 0.10342 & 30.2 & & & & 0.12728 & 32.0 \\
\hline & & 0.10455 & 30.2 & & & & 0.12867 & 32.0 \\
\hline & & 0.10565 & 30.0 & & & & 0.13004 & 31.9 \\
\hline & & 0.10673 & 30.1 & & & & 0.13139 & 31.8 \\
\hline & & 0.10779 & 30.1 & & & & 0.13271 & 32.0 \\
\hline & & 0.10884 & 30.1 & & & & 0.13400 & 31.8 \\
\hline & & 0.10986 & 30.1 & & & & 0.13528 & 31.9 \\
\hline & & 0.11087 & 30.1 & & & & & \\
\hline & & 0.11186 & 30.0 & & & & & \\
\hline & & 0.11283 & 30.1 & & & & & \\
\hline & & 0.11378 & 30.2 & & & & & \\
\hline & & 0.11472 & 30.0 & & & & & \\
\hline & & 0.11565 & 30.1 & & & & & \\
\hline & & 0.11655 & 30.2 & & & & & \\
\hline & & 0.11745 & 30.2 & & & & & \\
\hline & & 0.11832 & 30.2 & & & & & \\
\hline & & 0.11919 & 30.2 & & & & & \\
\hline & & 0.12003 & 30.1 & & & & & \\
\hline
\end{tabular}

Standard uncertainties are: $u(\mathrm{~T})=0.1 K, u(\mathrm{~m})=10^{-5} \mathrm{~m}, u(p)=0.05 p$ and $u(\gamma)=0.05 \gamma$. 